\title{
Strategy to reduce minimal magnetization switching field for Stoner particles
}

\author{
Z. Z. Sun and X. R. Wang \\ Physics Department, The Hong Kong University of Science and Technology, Clear Water Bay, Hong Kong SAR, China
}

(Received 7 July 2005; published 29 March 2006)

\begin{abstract}
A strategy is proposed aimed at substantially reducing the minimal magnetization switching field for a Stoner particle. Unlike the normal method of applying a static magnetic field which must be larger than the magnetic anisotropy, a much weaker field, proportional to the damping constant in the weak damping regime, can be used to switch the magnetization from one state to another if the field is along the motion of the magnetization. The concept is to constantly supply energy to the particle from the time-dependent magnetic field to allow the particle to climb over the potential barrier between the initial and the target states.
\end{abstract}

DOI: 10.1103/PhysRevB.73.092416

PACS number(s): 75.60.Jk, 75.75.+a, 85.70.Ay

\section{INTRODUCTION}

The recent advance in technology allows the fabrication of magnetic nanoparticles ${ }^{1,2}$ that are potentially useful for high density information storage. A magnetic nanoparticle, in which the magnetic moments of all atoms are aligned in the same direction, is called a Stoner particle. Manipulation of a Stoner particle ${ }^{3}$ is of significant interest in information processing. Finding an effective way to switch the magnetization from one state to another requires a clear understanding of magnetization dynamics. One important issue in magnetization reversal of Stoner particles is the minimal switching field. This problem was first studied by Stoner and Wohlfarth $^{4}$ (SW) who showed that a field $h$ larger than the SW-limit $h_{S W}$ can switch the magnetization from its initial state to the target value through a ringing effect. ${ }^{5-9}$ However, recent theoretical and experimental studies ${ }^{8-12}$ have shown that the minimal switching field can be smaller than the SW limit. Most studies have assumed the magnetic field to be time independent. However, a very recent experiment ${ }^{10}$ has shown that a dramatic reduction of the minimal field is possible by applying a small rf field pulse (the decrease in the constant field is much larger than the amplitude of the $\mathrm{rf}$ field). In this study, it has been shown that a small timedependent magnetic field can affect the magnetization of a Stoner particle such that the magnetization can move upward in its energy landscape against the dissipation effect. A consequence of this is that the minimal switching field is much smaller in comparison with the case of a time-independent magnetic field. In the case where the field magnitude does not change but the direction is allowed to vary, it can also be shown that the minimal field is proportional to the damping constant at the weak damping limit.

\section{DYNAMICS OF MAGNETIZATION IN A MAGNETIC FIELD}

The magnetization $\vec{M}=\vec{m} M_{s}$ of a Stoner particle can be conveniently described by a polar angle $\theta$ and an azimuthal angle $\phi$, shown in Fig. 1(a) where $\vec{m}$ is the unit direction of the magnetization, and $M_{s}$ is the saturated magnetization of the particle. In $\theta-\phi$ plane, each point corresponds to a particular state of the magnetization. The evolution of a state is governed by the Landau-Lifshitz-Gilbert (LLG) equation ${ }^{9,13}$

$$
\left(1+\alpha^{2}\right) \frac{d \vec{m}}{d t}=-\vec{m} \times \vec{h}_{t}-\alpha \vec{m} \times\left(\vec{m} \times \vec{h}_{t}\right),
$$

where $t$ is in a unit of $\left(|\gamma| M_{s}\right)^{-1}$, and the magnetic field is in the unit of $M_{s} .|\gamma|=2.21 \times 10^{5}(\mathrm{rad} / \mathrm{s}) /(\mathrm{A} / \mathrm{m})$ is the gyromagnetic ratio and $\alpha$ is a phenomenological dimensionless damping constant. The typical experimental values of $\alpha^{11}$ range from 0.037 to 0.22 for different Co films. The total field comes from an applied magnetic field $\vec{h}$ and the internal field $\vec{h}_{i}$ due to the magnetic anisotropy. Let $w(\vec{m}, \vec{h}=0)$ be the magnetic energy density function. Then $\vec{h}_{t}=-\nabla_{\vec{m}} w(\vec{m}, \vec{h}) / \mu_{0}$ $=\vec{h}_{i}+\vec{h}$, where $\mu_{0}=4 \pi \times 10^{-7} \mathrm{~N} / \mathrm{A}^{2}$ is the vacuum magnetic permeability. As shown in Fig. 1(a), the first term in the right-hand side (rhs) of Eq. (1) describes a precession motion around the total field and the second term is the damping motion toward the field.

The switching problem for a uniaxial Stoner particle is as follows: Before applying an external magnetic field, there are two stable fixed points [denoted by $A$ and $B$ in Fig. 1(b)] corresponding to magnetizations, say $\vec{m}_{0}$ (point $A$ ) and $-\vec{m}_{0}$ (point $B$ ) along its easy axis. The shadowed areas in Fig. 1(b) denote basins $A$ and $B$. The system in basin $A(B)$ will end up at state $A(B)$. Initially, the magnetization is $\vec{m}_{0}$, and the goal
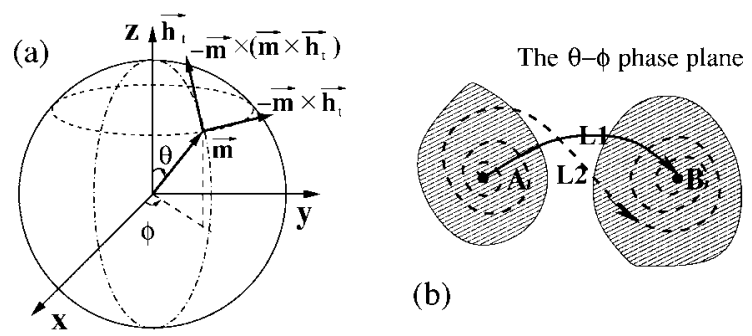

(b)

FIG. 1. (a) Two motions of magnetization $\vec{m}: \vec{h}_{t}$ is the total magnetic field. $-\vec{m} \times \vec{h}_{t}$ and $-\vec{m} \times\left(\vec{m} \times \vec{h}_{t}\right)$ describe the precession and dissipation motions, respectively. (b) The $\theta-\phi$ phase plane. Two stable fixed points $A$ and $B$ represent the initial and the target states, respectively. Two shadowed areas denote schematically basins of $A$ and $B$. The solid curve $L 1$ and dashed curve $L 2$ illustrate two different phase flows connected $A$ and $B$. 
is to reverse the magnetization to $-\vec{m}_{0}$ by applying an external field as small as possible. The issue is what is the minimal field $h_{c}$ defined as $h_{c}=\max \{h(t) ; \forall t\}$ for a given magnetic anisotropy.

\section{TIME-DEPENDENT VERSUS TIME-INDEPENDENT MAGNETIC FIELD}

In order to show that the magnetization reversal in a timedependent external magnetic field is qualitatively different from that in a constant field, it is useful to look at the energy change rate. From Eq. (1),

$$
\frac{d w}{d t}=-\frac{\alpha}{1+\alpha^{2}}\left(\vec{m} \times \vec{h}_{t}\right)^{2}-\vec{m} \cdot \dot{\vec{h}},
$$

where $\dot{\vec{h}}$ is the time derivative of $\vec{h}$. If the external field is time independent, the second term on the rhs vanishes, and hence, the energy will always decrease. In other words, a constant field is not an energy source. Conversely, a timedependent field can provide energy to a particle. According to Eq. (2), the second term on the rhs can be either positive or negative depending on the relative direction of $\vec{m}$ and $\dot{\vec{h}}$. This second term can even be larger than the first one so that the particle energy increases during its motion. $-\vec{m} \cdot \dot{\vec{h}}$ is a maximum when $\vec{m}$ and $\dot{\vec{h}}$ are in the opposite direction. From $|\vec{m}|=1$, it is known that $\vec{m}$ and $\dot{\vec{m}}$ are orthogonal to each other, which leads to $\vec{m} \cdot \ddot{\vec{m}}=-\dot{\vec{m}} \cdot \dot{\vec{m}}$. The second term on the rhs of Eq. (2) is the maximum when $\vec{h}=h_{0} \dot{\vec{m}} /|\dot{\vec{m}}|$ for a fixed $h_{0}$. Then, from Eqs. (1) and (2), the maximal rate of energy increase is

$$
\frac{d w}{d t}=\frac{\left|\vec{m} \times \vec{h}_{t}\right|}{\sqrt{1+\alpha^{2}}}\left(h_{0}-\frac{\alpha}{\sqrt{1+\alpha^{2}}}\left|\vec{m} \times \vec{h}_{t}\right|\right) .
$$

It should be highlighted that $\vec{h}$ is only well defined when $\dot{\vec{m}}$ $\neq 0$. Thus, in a numerical calculation, some numerical difficulties will exist when the system is near the extremes or the saddle points. Special care must be taken at these points.

\section{STRATEGY}

A strategy based on Eq. (3), can be developed using a smaller switching field. The field of magnitude $h_{0}$ noncollinear with the magnetization was applied to drive the system out of its initial minimum. Noncollinear fields have been proposed before in magnetization reversal. ${ }^{14}$ Fluctuations may also drive the system out of the minimum, but fluctuations are inefficient. When the system is out of the minimum and $\dot{\vec{m}} \neq 0$, a time-dependent field $\vec{h}=h_{0} \dot{\vec{m}} /|\dot{\vec{m}}|$ is applied such that $\dot{w}>0$. The system will climb the energy landscape from the bottom. When the system energy is very close to the saddle point, the field of magnitude $h_{0}$ can be rotated to noncollinear with the magnetization, say $\pi / 4$ to the direction of the target state so that problems at $\dot{\vec{m}}=0$ are avoided and the system can move closer to the target state. When the

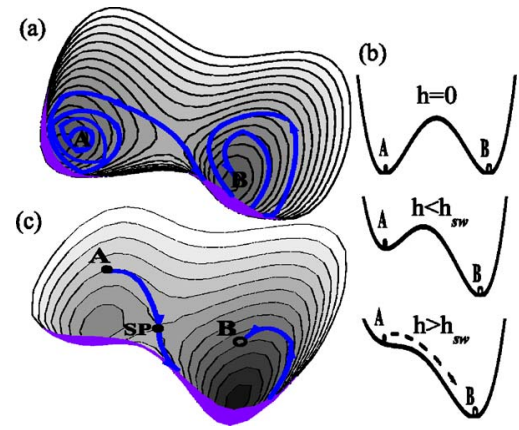

FIG. 2. (Color online) (a) Schematic illustration of the present strategy. The field along the motion of the magnetization provides the energy to the particle initially in the left energy minimum such that the system spins out of the minimum. Then a field opposite to the motion of the magnetization causes the system to spin into the right minimum. (b) The SW strategy: The target state (left minimum) becomes the only minimum when the magnetic field is larger than $h_{S W}$. The system will roll down along the potential landscape and end up at the target state. (c) The strategy in the precessional magnetization reversal: Both the initial (point $A$ ) and the target $(B)$ states are not local minima under the reversal field. Due to the magnetization dynamics described by the LLG equation, the particle will move along the trajectory denoted by the solid line on the energy landscape. The field is switched off as soon as the particle arrives at $B$.

system has overcome the potential barrier between the initial and target state and stays inside the basin of the target state, the field can be turned off or applied in the opposite direction to the motion of the magnetization, i.e., $\vec{h}=-h_{0} \dot{\vec{m}} /|\dot{\vec{m}}|$. In the first case, the system will reach the target state through the ringing motion caused by the energy dissipation, often due to the spin-lattice relaxation. In the second case, the system will move faster toward the target state because both terms on the rhs of Eq. (2) will be negative, resulting in a faster energy release from the particle.

The strategy is schematically illustrated in Fig. 2(a). The particle first spins out of its initial minimum by extracting energy from the field, and then spins into the target state by both energy dissipation and energy release (to the field). Since the energy gain from the field is partially compensated by the energy dissipation during the spinning-out process while both the field and the damping consume energy in the spinning-in motion, the particle moves out of its initial minimum slowly in comparison with its motion toward the target state. It can be readily seen in Fig. 2(a) that the particle makes more turns around the left minimum and fewer around the right minimum. A similar result was experimentally confirmed in Ref. 10. It will be shown later that a (linearly polarized) rf-field used in Ref. 10 is not the optimum. In fact, a circularly polarizedlike microwave (around $100 \mathrm{GHz}$ for a Co film ${ }^{11}$ ) is enough to switch a magnetization. The present strategy should be compared with those of the SW and the precessional pico-second magnetization reversal. ${ }^{11,12}$ As illustrated in Figs. 2(b) and 2(c), the SW strategy is to apply a large enough field to destroy the minimum initial state so that the particle can end up in the target state [Fig. 2(b)]. In the precessional magnetization reversal, ${ }^{9}$ the field is applied in 
such a way that the energy of the initial state is larger than that at the saddle point between the initial and final states. When the particle moves down the energy landscape, it will pass through the saddle point and arrive at the target state. The magnetization switch is achieved if the field is switched off at this point [Fig. 2(c)].

For simplicity, consider the case of an uniaxial magnetic anisotropy with the easy axis lying along the $x$ axis. The general form of $w(\vec{m}, \vec{h})$ can be written as

$$
w(\vec{m}, \vec{h})=-\mu_{0}\left(\frac{1}{2} k m_{x}^{2}+m_{x} h_{x}+m_{y} h_{y}+m_{z} h_{z}\right),
$$

where $h_{x}, h_{y}$, and $h_{z}$ are the applied magnetic fields along $x$, $y$, and $z$ axis, respectively. $k>0$ is the parameter measuring the strength of the anisotropy.

\section{RESULTS}

To find the minimal switching field for the uniaxial anisotropy of Eq. (4), it can be seen from Eq. (1), that $\dot{\vec{m}}$ is linear in the magnetic field, and as illustrated in Fig. 1(a), each field generates two motions for $\vec{m}$. The first is a precession around the field, and the second toward the field. Under the influence of the internal field (along the $x$ axis) and of the applied field $\vec{h}=h_{0} \dot{\vec{m}} /|\dot{\vec{m}}|$, the system evolves into a steady precession state for a small $h_{0}$ because the precession motion due to the applied field can exactly cancel the damping motion due to the internal field. The net motion (sum of precession around the internal field and damping motion due to the applied field) is a precession around the $x$ axis (easy axis). In this motion, the energy loss due to damping and the energy gain from the time-dependent external field are equal. The balance equation is

$$
h_{0}-k \alpha \cos \eta \sin \eta=0,
$$

where $\eta$ is the angle between the magnetization and the $x$ axis. The initial state is around $\eta=0$. Any stable precession

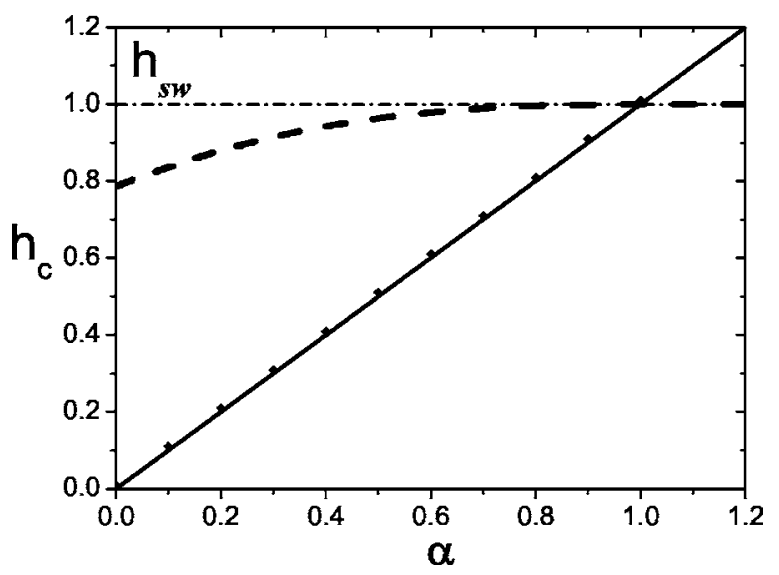

FIG. 3. The minimal reversal field (in unit $k / 2$ ) vs the damping constant. The diamond symbols are the numerical results of the present strategy for the uniaxial model of Eq. (4). The solid curve is the analytical results. For comparison, the dashed line is the minimal reversal field under a constant field $135^{\circ}$ to the $x$ axis for the same magnetic anisotropy.
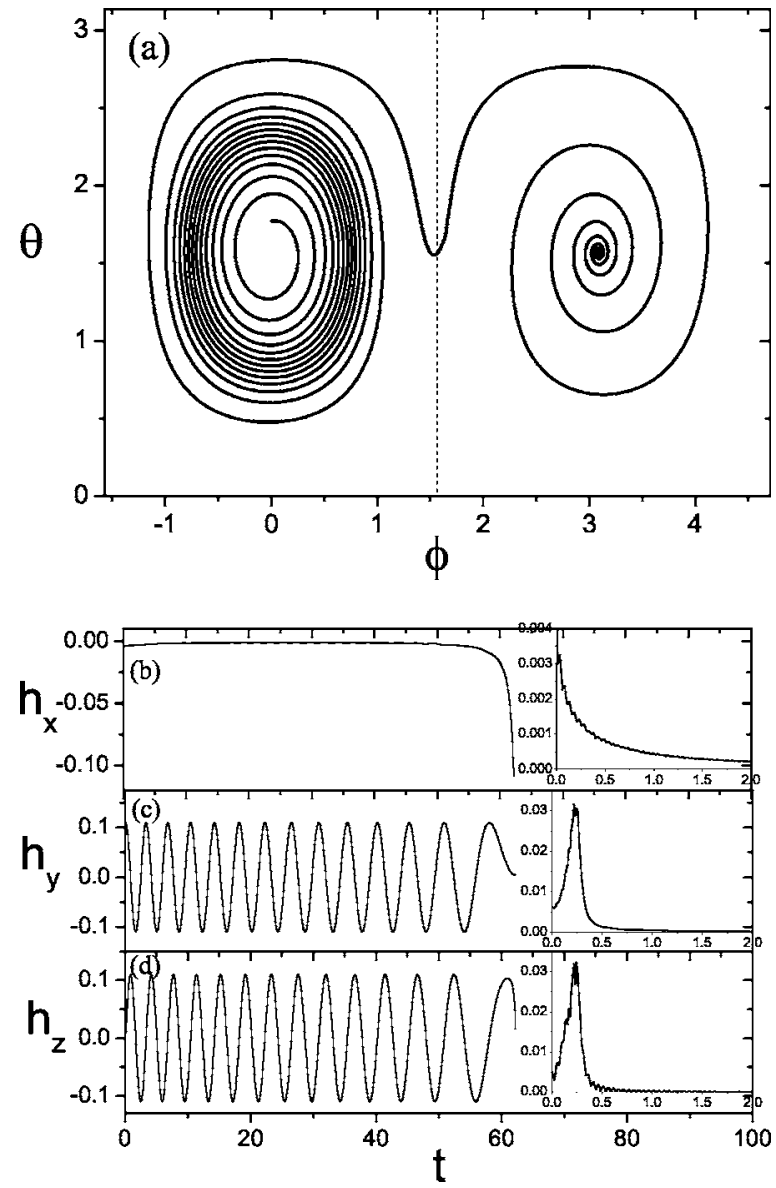

FIG. 4. (a) The phase flow under the present strategy with $k=2, \alpha=0.1$, and $h_{0}=0.11$. Just as illustrated in Fig. 2(a), the phase flow shows a slow spin-out motion near the initial state and a fast spin-in motion near the target state. (b)-(d) The time-dependent reversal field with the same parameter as that in (a). Insets: The corresponding Fourier transforms.

motion must be destroyed in order to push the system over the saddle point at $\eta=\pi / 2$. Since Eq. (5) has solutions only for $h_{0} \leqslant \alpha k / 2$, the critical field is

$$
h_{c}=\alpha k / 2 \text {. }
$$

It is of interest to note that the minimal reversal field is proportional to the damping constant, and approach zero when the damping constant goes to zero irrespective of how large the magnetic anisotropy is. For an arbitrary magnetic anisotropy, it may not be possible to find the analytical expression for the minimal reversal field, and should thus use numerical calculations. To demonstrate that this can indeed be done numerically, a calculation for the magnetic anisotropy of Eq. (4) has been performed. The result of the minimal reversal field versus damping constant $\alpha$ is plotted in Fig. 3. For comparison, the minimal reversal field for a timeindependent magnetic field laying at $135^{\circ}$ from the $x$ axis has been plotted. As it was explained in Ref. 9, the minimal reversal field is smaller than the SW limit for a small damping constant $\alpha<\alpha_{c}$ [which is 1 for the model given by Eq. (4)] and equals to the SW limit for $\alpha>\alpha_{c}$. It is clear that the 
present strategy is superior to that of SW or precessional reversal scheme only for $\alpha<1$, and is worse for larger $\alpha$.

To explain the type of field to be used in the current strategy, the trajectory of the system is numerically calculated and the time-dependent magnetic field is recorded. The results for $k=2, \alpha=0.1$, and $h_{0}=0.11>h_{c}$ are given in Fig. 4 . Figure $4(a)$ is the phase flow of the system starting from a point very close to the left minimum. As explained previously, the particle moves many turns in the left half of the phase plane before it crosses the potential barrier (the saddle point is on the middle line) while it moves toward the right minimum (the target state) much faster (with few turns). Figures $4(\mathrm{~b})-4(\mathrm{~d})$ are the corresponding time dependence of $x$, $y$, and $z$ components of the magnetic field. From these curves, it can be shown that $h_{y}$ and $h_{z}$ oscillate with time reflecting the spinning motion around minima. In general the spinning periods along different paths vary. Thus the timedependent magnetic field contains many different frequencies as can be seen from the Fourier transform of $h_{i}(t)$, $i=x, y, z$ shown in the insets of Figs. 4(b)-4(d). For Co-film parameters of $M_{s}=1.36 \times 10^{6} \mathrm{~A} / \mathrm{m},{ }^{11}$ the time unit is approximately $\left(|\gamma| M_{s}\right)^{-1}=3.33 \mathrm{ps}$. Correspondingly, the field consists of circularly-polarized microwaves of about $100 \mathrm{GHz}$.

\section{DISCUSSION AND CONCLUSIONS}

Although the switching field in the present scheme is much smaller than that in the old ones, it is an experimental challenge to create a time-dependent magnetic field required by this strategy. A device that is sensitive to the motion of the magnetization may be needed such that a coil can be attached to generate the required field. It should be emphasized that the results are based on the LLG equation which does not include any quantum effects. Quantum effects may be important for small particles ${ }^{15}$ whose level spacings are comparable with the energy quanta of the time-dependent field. In that case, a quantum version of LLG equation needs to be developed, which is beyond the scope of the present work.

In conclusion, a scheme is proposed to dramatically reduce the magnetization reversal field based on the fact that a time-dependent magnetic field can be both energy source and energy sink, depending on whether the field is parallel or anti-parallel to the motion of the magnetization. The idea is to constantly supply energy to a Stoner particle from the time-dependent magnetic field to allow the particle to move out of its initial minimum and to climb over the potential barrier. After the particle lands in the basin of the target state, the time-dependent field will act as an energy sink that constantly withdraw energy from the particle such that the particle will accelerate to the target state. In a simple model with an uniaxial magnetic anisotropy, the conditions and the solution of the steady precession motion in the present scheme for $h_{0}<h_{c}$ were also found.

\section{ACKNOWLEDGMENTS}

This work is supported by UGC, Hong Kong, through RGC CERG grants. X.R.W. would like to thank the hospitality of Laboratoire Pierre Aigrain, Ecole Normale Supérieure. Discussion with G. Bastard, P. Tong, and Ke Xia is acknowledged.
${ }^{1}$ S. Sun, C. B. Murray, D. Weller, L. Folks, and A. Moser, Science 287, 1989 (2000); D. Zitoun, M. Respaud, M.-C. Fromen, M. J. Casanove, P. Lecante, C. Amiens, and B. Chaudret, Phys. Rev. Lett. 89, 037203 (2002).

${ }^{2}$ M. H. Pan, H. Liu, J. Z. Wang, J. F. Jia, Q. K. Xue, J. L. Li, S. Qin, U. M. Mirdaidov, X. R. Wang, J. T. Market, Z. Y. Zhang, and C. K. Shih, Nano Lett. 5, 87 (2005).

${ }^{3}$ Spin Dynamics in Confined Magnetic Structures I \& II, edited by B. Hillebrands and K. Ounadjela (Springer-Verlag, Berlin, 2001).

${ }^{4}$ E. C. Stoner and E. P. Wohlfarth, Philos. Trans. R. Soc. London, Ser. A 240, 599 (1948).

${ }^{5}$ W. K. Hiebert, A. Stankiewicz, and M. R. Freeman, Phys. Rev. Lett. 79, 1134 (1997).

${ }^{6}$ Y. Acremann, C. H. Back, M. Buess, O. Portmann, A. Vaterlaus, D. Pescia, and H. Melchior, Science 290, 492 (2000).

${ }^{7}$ T. M. Crawford, T. J. Silva, C. W. Teplin, and C. T. Rogers, Appl. Phys. Lett. 74, 3386 (1999).

${ }^{8}$ L. He and W. D. Doyle, J. Appl. Phys. 79, 6489 (1996).
${ }^{9}$ Z. Z. Sun and X. R. Wang, Phys. Rev. B 71, 174430 (2005).

${ }^{10}$ C. Thirion, W. Wernsdorfer, and D. Mailly, Nat. Mater. 2, 524 (2003).

${ }^{11}$ C. H. Back, D. Weller, J. Heidmann, D. Mauri, D. Guarisco, E. L. Garwin, and H. C. Siegmann, Phys. Rev. Lett. 81, 3251 (1998); C. H. Back, R. Allenspach, W. Weber, S. S. P. Parkin, D. Weller, E. L. Garwin, and H. C. Siegmann, Science 285, 864 (1999).

${ }^{12}$ H. W. Schumacher, C. Chappert, P. Crozat, R. C. Sousa, P. P. Freitas, J. Miltat, J. Fassbender, and B. Hillebrands, Phys. Rev. Lett. 90, 017201 (2003); H. W. Schumacher, C. Chappert, R. C. Sousa, P. P. Freitas, and J. Miltat, ibid. 90, 017204 (2003).

${ }^{13}$ L. Landau and E. Lifshitz, Phys. Z. Sowjetunion 8, 153 (1953); T. L. Gilbert, Phys. Rev. 100, 1243 (1955).

${ }^{14}$ A. Brataas, Yu. V. Nazarov, and G. E. W. Bauer, Eur. Phys. J. B 22, 99 (2001); B. C. Choi, G. E. Ballentine, M. Belov, and M. R. Freeman, Phys. Rev. B 64, 144418 (2001).

${ }^{15}$ X. R. Wang and X. C. Xie, Europhys. Lett. 38, 55 (1997); X. R. Wang, S. C. Ma, and X. C. Xie, ibid. 45, 368 (1999). 